\title{
Design of a New Type of Transfer Device of Blowout Preventer and Fault Diagnosis and Analysis of the Rolling Bearing of Turbodrill
}

\author{
Wang Xu \\ Bohai Drilling Engineering Co., Ltd., Renqiu, Hebei, 062550 China \\ 178622407@qq.com
}

Keywords: transfer device; finite element analysis, mechanical model, blowout preventer unit

\begin{abstract}
The original installation method of the blowout preventer unit at the drilling site is time-consuming and unsafe, therefore, a new type of blowout preventer device is designed. The mechanical model of the device is set up, and the force condition of the hydraulic rods (the key components) of the blowout preventer unit is analyzed by finite element method. The conclusion is that the maximum force is relatively small in line with the allowable stress of the material, so it meets the safety requirement.
\end{abstract}

\section{Introduction}

With the continuous development of drilling technology, wells was deepening constantly, and the barometric pressure of underground oil was becoming increasingly high, the safety requirements for drilling wellhead blowout preventer was also increased, which makes the structure of the blowout preventer unit more complicated and heavy, and higher requirements on the installation of special equipment of the blowout preventer unit was also put forward.

\section{The establishment and analysis of mechanical model}

\subsection{The establishment of mechanical model}

The structure of the transfer device of the blowout preventer device is shown in figure 1 . The simplified mechanical model is shown in figure 2, and figure 3 is the force diagram of the scheme model. The variation of the maximum value of the force FA of the hydraulic rod is calculated by using the mechanical derivation and the simulation analysis of Matlab software. The calculation formula is as follows:

$$
\left.F_{A}=\frac{\frac{q 1 l_{1}^{2}}{2}+q_{2} l_{2}\left(l_{1}+\frac{l_{2}}{2}\right) \cos \alpha \sin \alpha \times}{\sqrt{\frac{h^{2}}{l_{1}^{2}}-2 \frac{h}{l_{1}} \cos \alpha+1}} \quad \text { (formula } 1\right)
$$

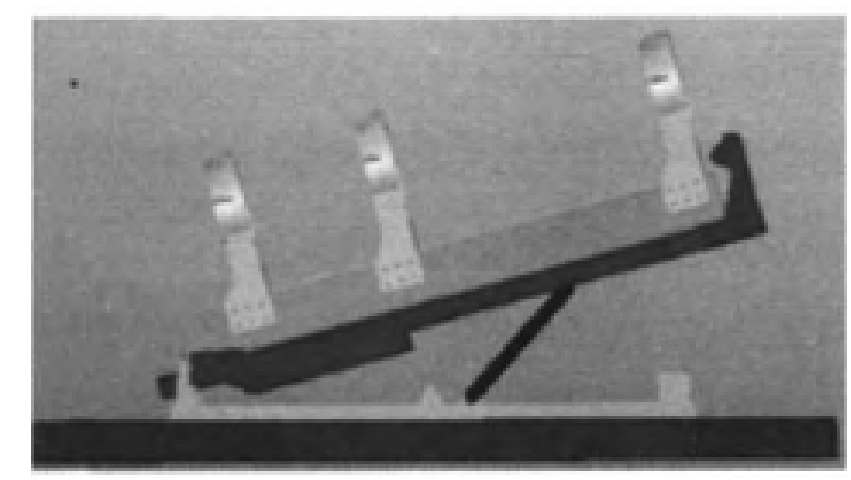

Figure 1 the structure of the transfer device of the blowout preventer 

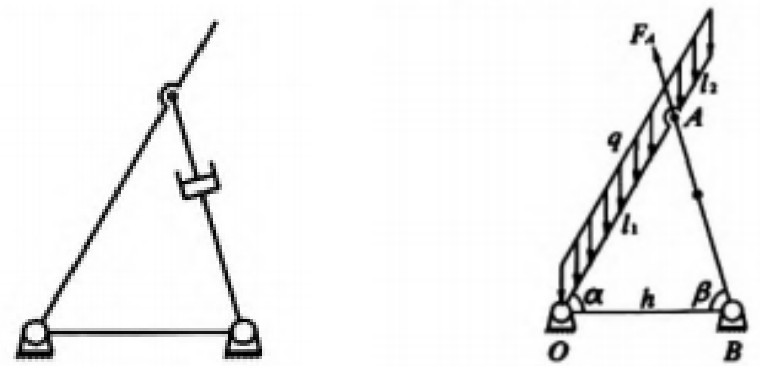

Figure 2 the simplified mechanical model Figure 3 the force diagram

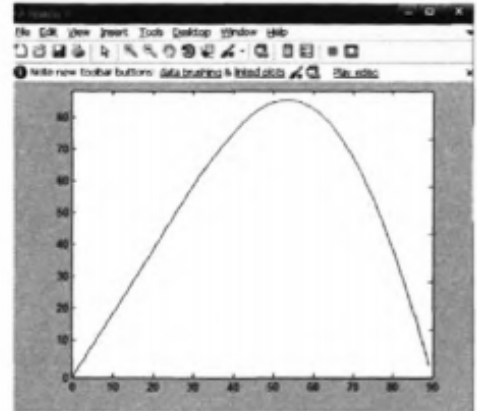

Figure 4 the change of the force of struts

Based on simulation analysis, the maximum value of FA is shown in Figure 4. The variation of the maximum force of the hydraulic rod is analyzed, and $n=52.5$ degree. FA gets the maximum, calculates $\mathrm{h}=2.4 \mathrm{~m}, \mathrm{l1}=3.2 \mathrm{~m}, \mathrm{l}=1.6 \mathrm{~m}, \mathrm{G}=150 \mathrm{kN}$, and $=0.966 \mathrm{G}$ in FA.

From the above analysis, we can see that the force of the hydraulic rod can meet the mechanical performance requirements of the material, and the telescopic motion of the hydraulic rod is relatively reasonable, which can not only save materials, but also ensure the stability of hydraulic poles.

\subsection{Overall size design of struts}

The whole struts has larger volume and larger bearing quality. Therefore, the 45 steel with better comprehensive properties is used as the main material, and all parts are welded by welding mode, and the bracket is connected through the pin shaft connection hinge. The hydraulic cylinder mainly promotes the movement of the struts in 3 directions, and hinges are also used to connect with the bracket. Three sets of hydraulic cylinders are needed to drive the whole bracket to move in 3 directions.

(1) The design of the base rail. The base need to bear the quality of all devices, the I-steel structure is used to achieve high stiffness and bending ability. The thickness of the steel is $15 \mathrm{~mm}$. The width of the base is $2200 \mathrm{~m}$ according to the width of the blowout preventer. The total length is 5729 m, and the structure is shown in Figure 5.
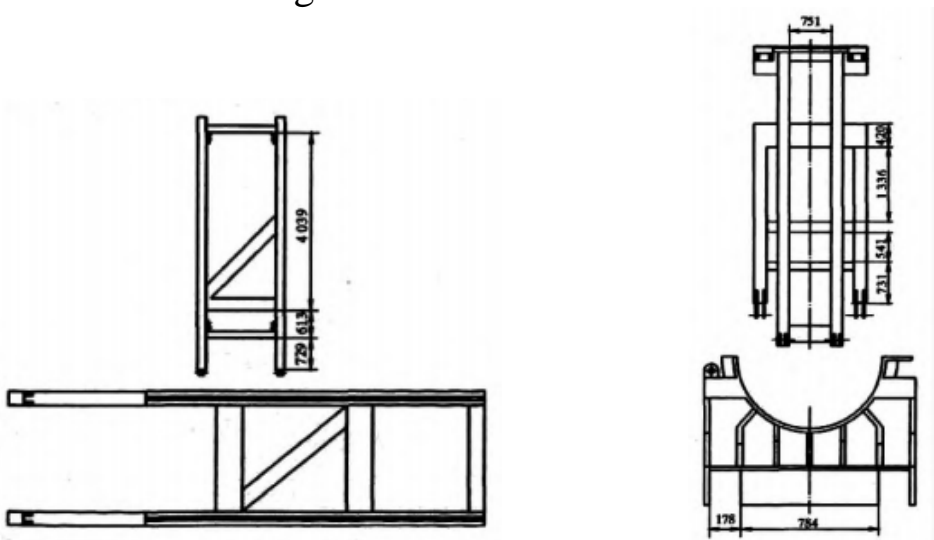

Figure 5 the structure of the base guide

Figure 6 the supported curtain and bracket 
The base guide mainly bears the entire device, moving forward and backward on the rail can be achieved among the horizontal guide, the upper base and the upper pulley, the structure of the main truss in the middle is used to increase the stability of the base, the left hinge is used to connect the upper struts of the hydraulic cylinder, so as to realize the movement of a device.

(2) The design of struts and bracket. Figure 6 is a schematic diagram of the struts and bracket structures. The struts connected with the base mainly bears the upper struts and are connected with the base through the hinge. The hydraulic cylinder for lifting is also connected with it, and the two hinge distance is $\mathrm{h}=2.4 \mathrm{~m}$. The middle beam is used to enhance the strength and stability of struts and support the upper struts, the wide groove is connected with the lower base to fixed struts, the narrow groove is connected with the hydraulic cylinder to make the blowout preventer move horizontally.

As the horizontal displacement is relatively small in order to maintain the balance movement of the whole struts, 4 hydraulic cylinders are used at the bottom, which are divided into 2 groups of parallel hydraulic cylinders. The struts mainly carries the E strut and three sets of blowout preventer. The width of the strut is $2000 \mathrm{~mm}$ according to the size of the blowout preventer, and the width of the middle strut is $800 \mathrm{~mm}$.

The blowout preventer struts are directly contacted with the blowout preventer group, so as to directly support the blowout preventer unit, and connect with the screw thread of the strut 3 , so that it is fixed on the top to support the total quality of the blowout preventer unit. The stiffened panels are increased mainly to improve the strength and endurance of struts, as well as the stability of the structure.

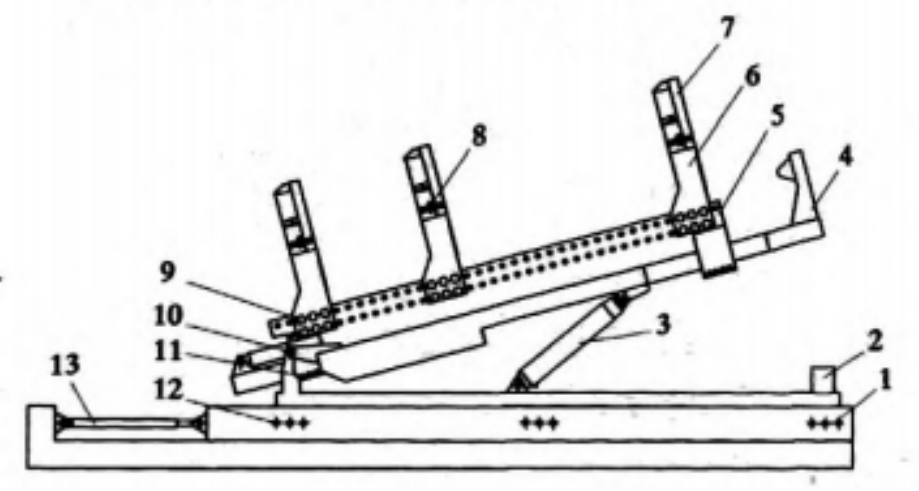

Figure 7 general assembly drawing of a blowout preventer

\section{Fault diagnosis and analysis of the rolling bearing of a turbodrill}

In rotating machinery, the failure of the bearing may cause the failure of the system. So far, there are many methods based on vibration to monitor the bearing state. The characteristics of the bearing vibration are seldom taken into account for these methods. The normal bearing system has been studied and analyzed, therefore the different types of bearing vibration have been made a new understanding. In the process of research, the contact between the roller and the raceway has been set as a nonlinear spring, and the system has been converted into a degree of freedom model. It is determined that the vibration characteristics of the inner ring basically depend on the inner clearance of the bearing. The periodic behavior of bearing failure thus produced will affect its chaotic behavior, and it can be reflected in Poincare map. 6 kinds of bearings with different types of faults are tested and their vibration signals are obtained.

Normal bearing.

For the fault of small inner ring, pits diameter is $1.1 \mathrm{~mm}$, damaged area is $0.95 \mathrm{~mm}^{2}$;

For the fault of large inner ring, damaged area is $4.1 \mathrm{~mm}^{2}$, as shown in Figure 8 (a);

For the fault small roller, pits diameter is $0.95 \mathrm{~mm}$, as shown in Figure 8 (b);

For the fault large roller, the diameter of the indentation is $2.1 \mathrm{~mm}$, as shown in Figure 8 (c);

For the fault of small outer ring, the local damaged area is $1.8 \mathrm{~mm}^{2}$;

For the fault of large outer ring, the local damaged area $3.1 \mathrm{~mm}^{2}$. 


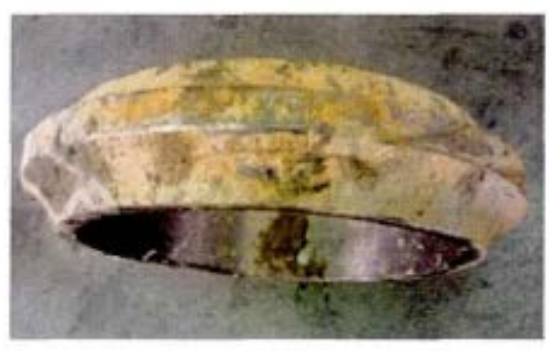

Figure 8 (a)

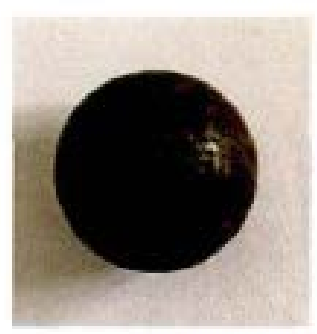

Figure 8 (b)

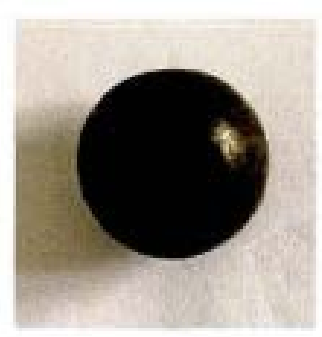

Figure 8 (c)

The bearings in the experiment are Roller bearings of Turbodrill, before the bearing string is assembled, every set of bearing is cleaned and lubricated, and running-in are made on the bearings 20 minutes before data collection, in order to compare the experimental results, each bearing fault are the same, the rotational speed is $600 \mathrm{rpm}$, the axial load is $23 \mathrm{kNo}$, the vibration signal is installed on the acceleration sensor of the bearing seat, which is measured by 3035AG, the accuracy was $100 \mathrm{~m} \mathrm{~V} / \mathrm{g}$. The vibration signals thus sampled are amplified and low-passing filtered by the 4105C. For high resonance frequency, the frequency of the central value of the passed filtering on the acquisition band is $24 \mathrm{kH}$. Through the above device, the sampling frequency of the vibration signal is set as $72 \mathrm{kHz}$. The anti-mixing filter is set as $30 \mathrm{kHz}$.

From the literature of existing fault diagnosis technology, the most commonly used monitoring methods include kurtosis, peak factor, envelope analysis, high-frequency resonance technology, Lyapunov exponent, continuous wavelet transform, discrete wavelet transform, correlation dimension and normalized information entropy. The diagnosis algorithm is compared, analyzed and realized by Matlab.

\subsection{Normalized information entropy}

According to the experimental data, the calculation of information entropy is much simpler than the maximum Lee Yap Andrian of index, and the increase of exponent will affect the calculation of information entropy. However, practically the vibration of bearing is more cyclical than that of low speed chaos. Therefore, if information entropy is used as the index of monitoring fault, the monitoring of high-speed system will be limited. In order to reduce the dependence of the index on the speed, the information entropy is normalized through the root mean square value of the signal. To calculate normalized information entropy and accelerate signals, the low channel filter is set as $2 \mathrm{kHz}$, and the data collected by analog to digital converter is $6 \mathrm{kH}$ : sampling frequency is digitalized.

\subsection{Kurtosis}

At different statistical moments, the vibration data is widely used as a monitoring indicator for bearing, which can be expressed as:

$$
M k=\int_{-\infty}^{+\infty}(x-\bar{x})^{k} d x
$$

(formula 2)

In formula 2, the $\mathrm{P}(\mathrm{x})$ is a probability distribution function, the average value is $\bar{x}$ on the $\mathrm{K}$ node of the data thus collected. Kurtosis, a numerical statistic reflecting the distribution characteristics of random variables, is normalized 4 order central moment $\left(\mathrm{RMS}^{4}\right)$. This index is usually used to measure the vibration peak caused by local bearing failure. If the bearing fault starts to diffuse within the bearing, then more peaks will be generated, and the root mean square value will also increase, thus the trend of the curve of kurtosis will be changed. In order to calculate kurtosis, the vibration signal is carried out at sampling frequency of $6000 \mathrm{~Hz}$. Then, data thus acquired is computed at low cut-off frequency of $1000 \mathrm{~Hz}$, and the formula is shown as 3 : 


$$
\text { Kurtosis }=\frac{\sum x^{4}}{\sigma^{2}}
$$

(formula 3)

\subsection{Peak factor}

Peak factor, or the range of values, is calculated by dividing the amplitude of the waveform with RMS. The peak factor has a strong ability to detect time domain signals with low energy. The peak factor is calculated by setting the original vibration signal and formula 4 with the frequency of $6000 \mathrm{~Hz}$.

$$
\text { Kurtosis }=\frac{\max (x)}{R M S(x)}
$$

(formula 4)

\subsection{Continuous wavelet transform}

The wavelet transform can be used as a tool to expand the data into different frequency components, and each component can be studied with the corresponding resolution. Since the wavelet transform technology can provide the information of the time domain signals and frequency signals, then it can also be used in the fault diagnosis technology.

\section{Conclusions}

The diagnosis system of bearing fault is proposed based on neural network. The above monitoring index is input as a diagnostic layer, and the output results correspond to the related working state or failure mode of the bearing. The contrastive analysis shows that the adaptive fuzzy neural inference system can map the working state of the bearing more effectively.

\section{References}

[1] Zhang Qiang, Chen Zhi, Luo Kaijia, Wen Juan, Dong Xiaohu. Research on 3D Cascade Design of Continuous Tube Small-sized Tturbodrill [J]. Science, Technology and Engineering, 2016,16 (07): 186-189.

[2] Tan Chunfei, Wang Yuxuan, Yao Yang, Li Bowen, Li Yang. Influence of Rotor Axial Clearance on Performance of Turbodrill [J]. Oil Field Equipment, 2016,45 (03): 7-10.

[3] Xu Fudong, Hu Chengfeng, Fu Daliang, von Ding. Development Status and Trend of Adjustable Turbodrill Reducer[J]. Journal of Jingchu Institute of Science and Technology, 2015,30 (06): 59-64.

[4] Tan Chunfei, Li Bowen, Wang Yuxuan, Yao Yang, Li can. Analysis and Calculation Method of Clamping Force for Fixed Rotor Installation of Turbodrill [J]. Oil Field Equipment, 2016,45 (04): 32-37.

[5] Zhao Liudong, Li Lixin, Wang Yu, Xue Qilong, Liu Baolin. Research Progress on Turbodrill [J]. Exploration Project (geotechnical drilling engineering), 2016,43 (10): 269-274. 